\title{
Borodin-Péché Fluctuations of the Free Energy in Directed Random Polymer Models
}

\author{
Zsófia Talyigás ${ }^{1} \cdot$ Bálint Vető ${ }^{2}$
}

Received: 4 October 2018 / Revised: 4 May 2019 / Published online: 23 May 2019

(c) The Author(s) 2019

\begin{abstract}
We consider two directed polymer models in the Kardar-Parisi-Zhang (KPZ) universality class: the O'Connell-Yor semi-discrete directed polymer with boundary sources and the continuum directed random polymer with $(m, n)$-spiked boundary perturbations. The free energy of the continuum polymer is the Hopf-Cole solution of the KPZ equation with the corresponding $(m, n)$-spiked initial condition. This new initial condition is constructed using two semi-discrete polymer models with independent bulk randomness and coupled boundary sources. We prove that the limiting fluctuations of the free energies rescaled by the $1 / 3$ rd power of time in both polymer models converge to the Borodin-Péché-type deformations of the GUE Tracy-Widom distribution.
\end{abstract}

\section{Introduction}

The Kardar-Parisi-Zhang (KPZ) equation was introduced for the description of physical surface growth phenomena in [14]. The equation gives the stochastic evolution of the height function $\mathcal{F}(T, X)$ where $T \in \mathbb{R}_{+}$is the time and $X \in \mathbb{R}$ is the space variable. It reads as

$$
\partial_{t} \mathcal{F}(T, X)=\frac{1}{2} \partial_{X}^{2} \mathcal{F}(T, X)+\frac{1}{2}\left(\partial_{X} \mathcal{F}(T, X)\right)^{2}+\xi(T, X), \quad \mathcal{F}(0, X)=\mathcal{F}_{0}(X)
$$

where $\xi$ denotes space-time Gaussian white noise with $\mathbb{E}[\xi(T, X) \xi(S, Y)]=\delta(T-$ $S) \delta(X-Y)$. By the presence of the nonlinear term, the equation is not rigorously

Bálint Vető

vetob@math.bme.hu

Zsófia Talyigás

z.talyigas@bath.ac.uk

1 Department of Mathematical Sciences, University of Bath, Claverton Down, Bath, UK

2 Institute of Mathematics, Budapest University of Technology and Economics, Egry J. u. 1, 1111 Budapest, Hungary 
well-posed and serious work is required to make sense of the solution directly [12]. A natural way to give a solution to the equation formally is via the stochastic heat equation (SHE) with multiplicative noise

$$
\partial_{T} \mathcal{Z}(T, X)=\frac{1}{2} \partial_{X}^{2} \mathcal{Z}(T, X)+\mathcal{Z}(T, X) \xi(T, X), \quad \mathcal{Z}(0, X)=\mathcal{Z}_{0}(X) .
$$

The latter equation is well-posed, and $\mathcal{F}(T, X)=\ln \mathcal{Z}(T, X)$ with initial condition $\mathcal{F}(0, X)=\ln \mathcal{Z}(0, X)$ defines a formal solution to (1.1) which is the Hopf-Cole solution of the KPZ equation. See [8] for a review on the KPZ equation and its universality class which is the family of models with the same scaling and asymptotic behaviour as the solution of the KPZ equation.

The Hopf-Cole solution of the KPZ equation can be understood as the partition function of a directed polymer model by the Feynman-Kac representation

$$
\mathcal{Z}(T, X)=\mathbb{E}_{B(X)}\left[\mathcal{Z}_{0}(B(0)): \exp :\left\{\int_{0}^{T} \xi(t, B(t)) d t\right\}\right]
$$

where the expectation $\mathbb{E}$ is taken over the law of a Brownian motion $B$ which is running backwards from time $T$ and position $X$ and where : exp : is the Wick exponential. The representation (1.3) defines the partition function of the continuum directed random polymer (CDRP) as it is the total weight of Brownian paths where the weight is proportional to the exponential function of the integral of the disorder along the path. The logarithm of the partition function $\mathcal{F}(T, X)=\ln \mathcal{Z}(T, X)$ is called the free energy of the CDRP.

The present paper describes limiting fluctuations in two directed polymer models. Directed polymers are well-studied objects in the KPZ universality class of models in the recent mathematics and physics literature. The reason for the special interest is that certain models possess exact solvable properties, i.e. explicit formulas can be derived for some of their important observables. The first directed polymer model with exact solvability is the O'Connell-Yor semi-discrete polymer [17,19]. Exactly, solvable polymers on the square lattice are the log-gamma polymer [9,20,21], the strict-weak polymer [10,18], the beta polymer [3] and the inverse beta polymer [22]. Methods to obtain exact solvability include explicit stationary measure, Bethe Ansatz integrability and the (geometric) Robinson-Schensted-Knuth (RSK) correspondence.

In [5], the O'Connell-Yor model was considered with boundary perturbations. The large time limit of the free energy was proved to be the Baik-Ben Arous-Péché (BBP) distribution [2] which is the perturbed version of the GUE Tracy-Widom distribution. A similar limit distribution was obtained for the CDRP with $m$-spiked boundary perturbation in [5].

The results of the present paper generalize those of [5] in the following sense. We investigate the large scale behaviour of the free energy of two directed polymer models. The first model is the O'Connell-Yor semi-discrete random polymer with loggamma boundary sources [6] which is the mixture of the O'Connell-Yor semi-discrete polymer with boundary perturbations considered in [5] and the log-gamma discrete directed polymer. Explicit Fredholm determinant expressions are available in [6] for the Laplace transform of the partition function of the polymer mixture model. Based 
on these formulas, we obtain the single time version of the Borodin-Péché distribution as the limit distribution of the free energy. The Borodin-Péché distribution which is a generalization of the BBP distribution was first described in its multi-time version in last passage percolation with defective rows and columns and in a single time version in a random matrix model in [7].

A closely related model is the stationary O'Connell-Yor polymer model which was considered in [13] as the limit of the O'Connell-Yor semi-discrete polymer model with log-gamma boundary sources. It was proved in [13] that the large time limit of the stationary model is the Baik-Rains distribution and the solution of the stationary KPZ equation was obtained as the scaling limit of the stationary O'Connell-Yor polymer.

The second model considered in the present paper is the CDRP which can be obtained as the limit of the O'Connell-Yor semi-discrete polymer under the intermediate disorder scaling $[11,16]$. Extending the investigations of the CDRP with $m$-spiked boundary perturbation in [5], we introduce the $(m, n)$-spiked boundary perturbation. The $m$-spiked boundary perturbation is nonzero for the positive values of the space variable, and the $(m, n)$-spiked boundary perturbation can be seen as its two-sided version with the appropriate coupling of the two sides. We prove Borodin-Péché limit distribution for the free energy of the CDRP with $(m, n)$-spiked boundary perturbation based on explicit Fredholm determinant formulas from [6].

The rest of the paper is organized as follows. We introduce the O'Connell-Yor semidiscrete directed random polymer with log-gamma boundary sources and the CDRP with $(m, n)$-spiked boundary perturbation in Sect. 2. Our main results, Theorem 2.3 and Theorem 2.5 are also stated in this section. We prove Theorem 2.3 in Sect. 3 and Theorem 2.5 in Sect. 4.

\section{Models and Main Results}

We present the two models considered in this paper: the O'Connell-Yor semi-discrete directed polymer with log-gamma boundary sources and the continuum directed random polymer (CDRP) with $(m, n)$-spiked boundary perturbation. These models were defined in [6], but the $(m, n)$-spiked boundary perturbation is new. We consider a slightly different scaling of the boundary perturbations as in [6] yielding our main results which are stated in this section.

\subsection{O'Connell-Yor Semi-discrete Directed Polymer with Log-Gamma Boundary Sources}

The O'Connell-Yor semi-discrete directed polymer with log-gamma boundary sources is the mixture of the semi-discrete polymer model introduced by O'Connell and Yor [19] and the discrete one by Seppäläinen [20]. By log-gamma distribution with parame$\operatorname{ter} \theta>0$, we mean the distribution of the random variable $-\ln X$ where $X$ has gamma distribution with parameter $\theta$, i.e. when $X$ has density $x^{\theta-1} e^{-x} / \Gamma(\theta)$ for $x>0$.

Fix $N \geq 1$ and $n \geq 0$. Let $a=\left(a_{1}, \ldots, a_{N}\right) \in \mathbb{R}^{N}$ and $\alpha=\left(\alpha_{1}, \ldots, \alpha_{n}\right) \in \mathbb{R}_{+}^{n}$ be such that $\alpha_{k}-a_{l}>0$ for all $1 \leq l \leq N$ and $1 \leq k \leq n$. In the polymer model that we introduce, the horizontal axis is discrete on the left of 0 and continuous on the right 


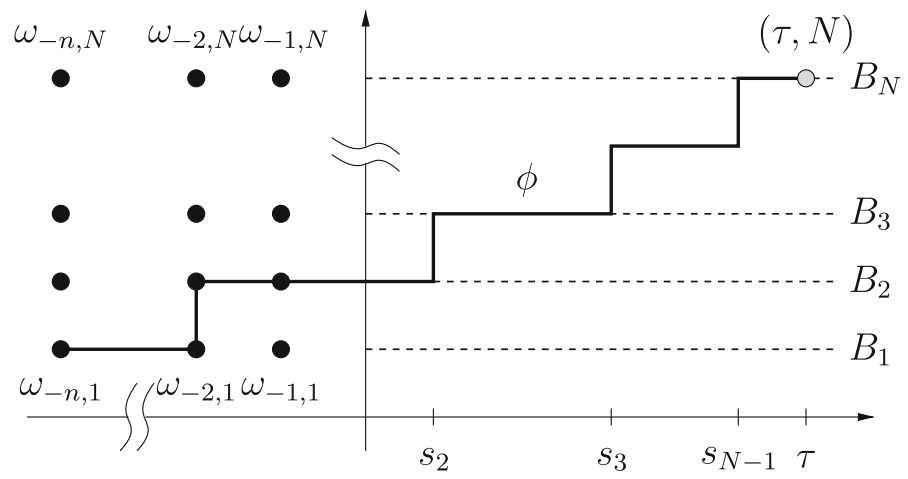

Fig. 1 O'Connell-Yor semi-discrete directed polymer with log-gamma boundary sources. The thick solid line is a possible path $\phi$ from $(-n, 1)$ to $(\tau, N)$. The random variables $\omega_{-k, l}$ have log-gamma distribution with parameter $\alpha_{k}-a_{l}$, and the Brownian motions $B_{1}, \ldots, B_{N}$ have drifts $a_{1}, \ldots, a_{N}$

of 0 while the vertical axis is discrete. For all $1 \leq k \leq n$ and $1 \leq l \leq N$, let $\omega_{-k, l}$ be independent log-gamma random variables with parameter $\alpha_{k}-a_{l}$. For all $1 \leq l \leq N$, let $B_{l}$ be independent Brownian motions with drift $a_{l}$ which are also independent of the log-gamma variables. The $\omega_{-k, l}$ can be thought of as sitting at the lattice points $(-k, l)$, while $B_{l}$ can be thought of as sitting along the horizontal ray from $(0, l)$ as shown on Fig. 1.

Admissible paths consist of discrete and semi-discrete parts. A discrete upright path $\phi^{d}:\left(i_{1}, j_{1}\right) \nearrow\left(i_{\ell}, j_{\ell}\right)$ is an ordered set of points $\left(\left(i_{1}, j_{1}\right),\left(i_{2}, j_{2}\right), \ldots,\left(i_{\ell}, j_{\ell}\right)\right)$ with each $\left(i_{k}, j_{k}\right) \in \mathbb{Z}^{2}$ and each increment $\left(i_{k}, j_{k}\right)-\left(i_{k-1}, j_{k-1}\right)$ either $(1,0)$ or $(0,1)$. A semi-discrete upright path $\phi^{s d}:(0, l) \nearrow(\tau, N)$ is a union of horizontal line segments $\left((0, l) \rightarrow\left(s_{l}, l\right)\right) \cup\left(\left(s_{l}, l+1\right) \rightarrow\left(s_{l+1}, l+1\right)\right) \cup \cdots \cup\left(\left(s_{N-1}, N\right) \rightarrow(\tau, N)\right)$ where $0 \leq s_{l}<s_{l+1}<\cdots<s_{N-1} \leq \tau$. It is convenient to think of $\phi^{s d}$ as a surjective non-decreasing function from $[0, \tau]$ onto $\{l, \ldots, N\}$. Our upright paths $\phi$ in the mixture model are composed of discrete portions $\phi^{d}$ adjoined to semi-discrete portions $\phi^{s d}$ in such a way that for some $1 \leq l \leq N, \phi^{d}:(-n, 1) \nearrow(-1, l)$ and $\phi^{s d}:(0, l) \nearrow(\tau, N)$.

To an upright path described above, we associate an energy

$$
E(\phi)=\sum_{(i, j) \in \phi^{d}} \omega_{i, j}+B_{l}\left(s_{l}\right)+\left(B_{l+1}\left(s_{l+1}\right)-B_{l+1}\left(s_{l}\right)\right)+\cdots+\left(B_{N}(\tau)-B_{N}\left(s_{N-1}\right)\right)
$$

which aggregates the randomness along the path, and hence, itself is random depending on $\omega_{i, j}$ and $B_{1}, \ldots, B_{N}$. The polymer measure on a path $\phi$ is proportional to its Boltzmann weight given by $e^{E(\phi)}$. The normalizing constant or polymer partition function for the O'Connell-Yor semi-discrete directed polymer with log-gamma boundary sources is the integral of the Boltzmann weight over the background measure on the path space $\phi$, i.e.

$$
\mathbf{Z}^{a, \alpha}(\tau, N)=\sum_{l=1}^{N} \sum_{\phi^{d}:(-n, 1) \nearrow(-1, l)} \int_{\phi^{s d}:(0, l) \nearrow(\tau, N)} e^{E(\phi)} \mathrm{d} \phi^{s d}
$$


where $\mathrm{d} \phi^{s d}$ represents the Lebesgue measure on the simplex $0 \leq s_{k}<s_{k+1}<$ $\cdots<s_{N-1} \leq \tau$ with which $\phi^{s d}$ is identified. The free energy of the O'Connell-Yor semi-discrete directed polymer with log-gamma boundary sources is given by

$$
\mathbf{F}^{a, \alpha}(\tau, N)=\ln \left(\mathbf{Z}^{a, \alpha}(\tau, N)\right)
$$

The distribution of the partition function $\mathbf{Z}^{a, \alpha}(\tau, N)$ of the O'Connell-Yor semidiscrete directed polymer with log-gamma boundary sources is characterized in [6] as follows.

Theorem $2.1\left[6\right.$, Theorem 2.1] Fix $N \geq 9, n \geq 0$ and $\tau>0$. Let $a=\left(a_{1}, \ldots, a_{N}\right) \in$ $\mathbb{R}^{N}$ and $\alpha=\left(\alpha_{1}, \ldots, \alpha_{n}\right) \in \mathbb{R}_{+}^{n}$ be such that $\alpha_{k}-a_{l}>0$ for all $1 \leq l \leq N$ and $1 \leq k \leq n$. For $1 \leq k \leq n$ and $1 \leq l \leq N$ let $\omega_{-k, l}$ be independent log-gamma random variables with parameter $\alpha_{k}-a_{l}$ and for all $1 \leq l \leq N$ let $B_{l}$ be independent Brownian motions with drift $a_{l}$. Then, for all $u \in \mathbb{C}$ with positive real part

$$
\mathbb{E}\left(e^{-u \mathbf{Z}^{a, \alpha}(\tau, N)}\right)=\operatorname{det}\left(\mathbb{1}+\mathrm{K}_{u}\right)_{L^{2}\left(\mathcal{C}_{a ; \alpha ; \varphi}\right)}
$$

where the operator $\mathrm{K}_{u}$ is defined in terms of its integral kernel

$$
\begin{aligned}
\mathrm{K}_{u}\left(v, v^{\prime}\right)= & \frac{1}{2 \pi \mathrm{i}} \int_{\mathcal{D}_{v}} \mathrm{~d} s \Gamma(-s) \Gamma(1+s) \frac{u^{s} e^{v \tau s+\tau s^{2} / 2}}{v+s-v^{\prime}} \prod_{l=1}^{N} \frac{\Gamma\left(v-a_{l}\right)}{\Gamma\left(s+v-a_{l}\right)} \\
& \times \prod_{k=1}^{n} \frac{\Gamma\left(\alpha_{k}-v-s\right)}{\Gamma\left(\alpha_{k}-v\right)} .
\end{aligned}
$$

The contours $\mathcal{C}_{a ; \alpha ; \varphi}$ and $\mathcal{D}_{v}$ are given in Definition 2.2 below where $\varphi \in(0, \pi / 4)$ is arbitrary.

Definition 2.2 Let $a=\left(a_{1}, \ldots, a_{N}\right) \in \mathbb{R}^{N}$ and $\alpha=\left(\alpha_{1}, \ldots, \alpha_{n}\right) \in \mathbb{R}_{+}^{n}$ be such that $\alpha_{k}-a_{l}>0$ for all $1 \leq l \leq N$ and $1 \leq k \leq n$. Set $\mu=\frac{1}{2} \max (a)+\frac{1}{2} \min (\alpha)$ and $\eta=\frac{1}{4} \max (a)+\frac{3}{4} \min (\alpha)$. Then, for all $\varphi \in(0, \pi / 4)$, we define the contour $\mathcal{C}_{a ; \alpha ; \varphi}=\left\{\mu+e^{\mathrm{i}(\pi+\varphi)} y\right\}_{y \in \mathbb{R}_{+}} \cup\left\{\mu+e^{\mathrm{i}(\pi-\varphi)} y\right\}_{y \in \mathbb{R}_{+}}$. The contour is oriented so as to have increasing imaginary part. For every $v \in \mathcal{C}_{a ; \alpha ; \varphi}$, we choose $R=-\operatorname{Re}(v)+\eta$, $d>0$, and define a contour $\mathcal{D}_{v}$ as follows. $\mathcal{D}_{v}$ goes by straight lines from $R-\mathrm{i} \infty$, to $R-\mathrm{i} d$, to $1 / 2-\mathrm{i} d$, to $1 / 2+\mathrm{i} d$, to $R+\mathrm{i} d$, to $R+\mathrm{i} \infty$. The parameter $d$ is taken small enough so that $v+\mathcal{D}_{v}$ does not intersect $\mathcal{C}_{a ; \alpha ; \varphi}$. See Fig. 2 for an illustration.

Our contribution on the O'Connell-Yor semi-discrete directed polymer with loggamma boundary sources is that we prove a Borodin-Péché scaling limit of its free energy. To define the limiting distribution, fix two integers $m$ and $n$. Let $b=\left(b_{1}, \ldots, b_{m}\right) \in \mathbb{R}^{m}$ and $\beta=\left(\beta_{1}, \ldots, \beta_{n}\right) \in \mathbb{R}^{n}$ be two sets of parameters and assume that

$$
\max _{1 \leq l \leq m} b_{l}<\min _{1 \leq k \leq n} \beta_{k}
$$



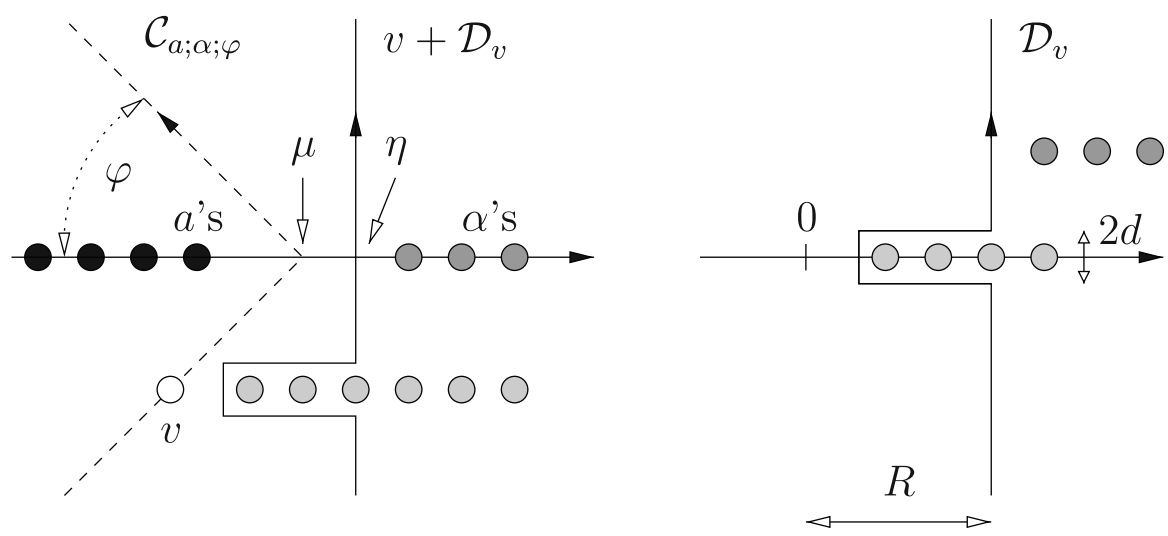

Fig. 2 Left: the contour $\mathcal{C}_{a ; \alpha ; \varphi}$ (dashed) where the black dots symbolize the set of singularities of $K_{u}\left(v, v^{\prime}\right)$ in $v$ at $\cup_{1 \leq l \leq N}\left\{a_{l}, a_{l}-1, \ldots\right\}$ coming from the factors $\Gamma\left(v-a_{l}\right)$. The contour $v+\mathcal{D}_{v}$ is the solid line. Right: the contour $\mathcal{D}_{v}$ where the light grey dots are the singularities at $\{1,2, \ldots\}$ coming from $\Gamma(-s)$ and the dark grey dots are those at $\cup_{1 \leq k \leq n}\left\{\alpha_{k}-v, \alpha_{k}+1-v, \ldots\right\}$ coming from $\Gamma\left(\alpha_{k}-v-s\right)$

which is a natural constraint, since otherwise the corresponding polymer models are not well defined, see Theorems 2.3 and 2.5 for the parameter scaling.

The Borodin-Péché distribution with parameters $b$ and $\beta$ [7] is defined as

$$
F_{\mathrm{BP}, b, \beta}(r)=\operatorname{det}\left(\mathbb{1}-\mathrm{K}_{\mathrm{BP}, b, \beta}\right)_{L^{2}((r, \infty))}
$$

with the kernel

$$
\mathrm{K}_{\mathrm{BP}, b, \beta}(x, y)=\frac{1}{(2 \pi \mathrm{i})^{2}} \int_{\gamma} \mathrm{d} w \int_{\Gamma} \mathrm{d} z \frac{1}{z-w} \frac{e^{z^{3} / 3-z y}}{e^{w^{3} / 3-w x}} \prod_{l=1}^{m} \frac{z-b_{l}}{w-b_{l}} \prod_{k=1}^{n} \frac{w-\beta_{k}}{z-\beta_{k}}
$$

where the integration contours $\gamma$ and $\Gamma$ are given as follows. Let $c>0$ be arbitrary. Then, $\gamma$ is $-c+\mathrm{i} \mathbb{R}$ modified in a neighbourhood of the real axis so that it crosses the axis between $\max _{1 \leq l \leq m} b_{l}$ and $\min _{1 \leq k \leq n} \beta_{k}$. The contour $\Gamma$ is $c+\mathrm{i} \mathbb{R}$ modified in a neighbourhood of the real axis so that it crosses the real axis between $\max _{1 \leq l \leq m} b_{l}$ and $\min _{1 \leq k \leq n} \beta_{k}$, and it does not intersect $\gamma$. We mention that for $n=0$, the BorodinPéché distribution reduces to the BBP distribution and for $n=m=0$ to the GUE Tracy-Widom distribution.

To state our main theorem on the scaling limit of the O'Connell-Yor semi-discrete directed polymer with log-gamma boundary sources, we will use the following parametrization. Let $\Psi(z)=\frac{d}{d z} \ln \Gamma(z)$ be the digamma function. For a given $\theta \in \mathbb{R}_{+}$, define

$$
\kappa(\theta)=\Psi^{\prime}(\theta), \quad f(\theta)=\theta \Psi^{\prime}(\theta)-\Psi(\theta), \quad c(\theta)=\left(-\Psi^{\prime \prime}(\theta) / 2\right)^{1 / 3} .
$$

We may alternatively parameterize $\theta \in \mathbb{R}_{+}$in terms of $\kappa \in \mathbb{R}_{+}$as

$$
\theta_{\kappa}=\left(\Psi^{\prime}\right)^{-1}(\kappa) \in \mathbb{R}_{+}, \quad f_{\kappa}=\inf _{t>0}(\kappa t-\Psi(t))=f\left(\theta_{\kappa}\right), \quad c_{\kappa}=c\left(\theta_{\kappa}\right) .
$$


Theorem 2.3 Consider the O'Connell-Yor semi-discrete directed random polymer with log-gamma boundary sources of the following parameters. Let $a=$ $\left(a_{1}, a_{2}, \ldots, a_{m}, 0, \ldots 0\right) \in \mathbb{R}^{N}$ with $m \leq N$ and $\alpha=\left(\alpha_{1}, \alpha_{2}, \ldots, \alpha_{n}\right) \in \mathbb{R}^{n}$ where $a_{1}, a_{2}, \ldots a_{m}$ may depend on $N$ and $\alpha_{k}>\max _{1 \leq l \leq m} a_{l}$ for $1 \leq k \leq n$. Let $\kappa>0$ be arbitrary. Assume furthermore that there are real parameters $b=\left(b_{1}, \ldots, b_{m}\right)$ and $\beta=\left(\beta_{1}, \ldots \beta_{n}\right)$ satisfying (2.6) such that for any $1 \leq l \leq m$ and $1 \leq k \leq n$,

$$
\lim _{N \rightarrow \infty} c_{\kappa} N^{1 / 3}\left(a_{l}(N)-\theta_{\kappa}\right)=b_{l} \text { and } \lim _{N \rightarrow \infty} c_{\kappa} N^{1 / 3}\left(\alpha_{k}(N)-\theta_{\kappa}\right)=\beta_{k} .
$$

Then,

$$
\lim _{N \rightarrow \infty} \mathbb{P}\left(\frac{\mathbf{F}^{a, \alpha}(\kappa N, N)-N f_{\kappa}}{c_{\kappa} N^{1 / 3}} \leq r\right)=F_{\mathrm{BP}, b, \beta}(r)
$$

holds where $\mathbf{F}^{a, \alpha}$ is the free energy of the O'Connell-Yor semi-discrete directed random polymer given in (2.3) and $F_{\mathrm{BP}, b, \beta}$ is the Borodin-Péché distribution function defined in (2.7).

\subsection{Continuum Directed Random Polymer (CDRP) with $(m, n)$-Spiked Boundary Perturbation}

The partition function $\mathcal{Z}(T, X)$ of the continuum directed random polymer with boundary perturbation $\mathcal{Z}_{0}(X)$ is given by the solution to the stochastic heat equation with multiplicative noise (1.2) with initial condition $\mathcal{Z}_{0}(X)$. The initial data $\mathcal{Z}_{0}(X)$ may be random, but it is assumed to be independent of the space-time white noise.

By the Feynman-Kac representation $(1.3), \mathcal{Z}(T, X)$ is indeed a partition function of a directed polymer model, since Brownian paths are reweighted in a way that the weight of a path is proportional to the Wick exponential of the randomness integrated along the path. The normalizing constant which is the partition function $\mathcal{Z}(T, X)$ is the integral of weights over the space of all possible paths. Note that $\mathcal{Z}(T, X)$ itself is random as the randomness of the space-time white noise remains in the formula (1.3).

By the work of Mueller [15], as long as $\mathcal{Z}_{0}(X)$ is almost surely positive, $\mathcal{Z}(T, X)$ is positive for all $T>0$ and $X \in \mathbb{R}$ almost surely. Hence, we can take its logarithm and define the free energy for the continuum directed random polymer with boundary perturbation $\ln \mathcal{Z}_{0}(X)$ by $\mathcal{F}(T, X)=\ln (\mathcal{Z}(T, X))$ to be the Hopf-Cole solution of the KPZ equation (1.1) with initial condition $\mathcal{F}_{0}(X)=\ln \mathcal{Z}_{0}(X)$.

Let us now introduce the CDRP with $(m, n)$-spiked boundary perturbation, and let us construct the corresponding $(m, n)$-spiked initial condition for the stochastic heat equation. For fixed integers $m$ and $n$, let $b=\left(b_{1}, \ldots, b_{m}\right) \in \mathbb{R}^{m}$ and $\beta=\left(\beta_{1}, \ldots, \beta_{n}\right) \in \mathbb{R}^{n}$ be such that (2.6) holds. Let $B_{1}, B_{2}, \ldots, B_{m}$ be independent Brownian motions with drifts $b_{1}, b_{2}, \ldots, b_{m}$, and let $\widetilde{B}_{1}, \widetilde{B}_{2}, \ldots, \widetilde{B}_{n}$ be independent Brownian motions with drifts $\beta_{1}, \beta_{2}, \ldots, \beta_{n}$. Furthermore, let $\omega_{-k, l}$ be independent $\log$-gamma random variables with parameter $\beta_{k}-b_{l}$ for $1 \leq l \leq m$ and $1 \leq k \leq n$. Assume that the two families of Brownian motions and the log-gamma random vari- 
ables are independent of each other. For $X \geq 0$, let the semi-discrete partition function $\mathbf{Z}^{b, \beta}(X, m)$ be constructed as in (2.2) using the Brownian motions $B_{1}, B_{2}, \ldots, B_{m}$ and the log-gamma random variables.

Similarly, we construct another semi-discrete partition function which is coupled to the previous one. Let the possible paths $\widetilde{\phi}$ be composed of a discrete upright part $\widetilde{\phi}^{d}:(-n, 1) \nearrow(k-n-1, m)$ and of a semi-discrete part $\widetilde{\phi}^{s d}$. For $\widetilde{X} \geq 0$, let the semi-discrete part $\widetilde{\phi}^{s d}:(k-n-1, m) \nearrow(-1, \widetilde{X})$ be a union of vertical line segments $\left((k-n-1, m) \rightarrow\left(k-n-1, s_{k}\right)\right) \cup\left(\left(k-n, s_{k}\right) \rightarrow\left(k-n, s_{k+1}\right)\right) \cup \cdots \cup\left(\left(-1, s_{n-1}\right) \rightarrow\right.$ $(-1, \tilde{X}))$ where $m \leq s_{k}<s_{k+1}<\cdots<s_{n-1} \leq \tilde{X}$. The energy of such a path is instead of (2.1) defined by

$$
\begin{aligned}
E(\widetilde{\phi})= & \sum_{(i, j) \in \widetilde{\phi}^{d}} \omega_{i, j}+\widetilde{B}_{k}\left(s_{k}\right)+\left(\widetilde{B}_{k+1}\left(s_{k+1}\right)-\widetilde{B}_{k+1}\left(s_{k}\right)\right)+\cdots+\left(\widetilde{B}_{n}(\widetilde{X})\right. \\
& \left.-\widetilde{B}_{n}\left(s_{n-1}\right)\right) .
\end{aligned}
$$

Then, a partition function analogously to (2.2) is given by

$$
\widetilde{\mathbf{Z}}^{b, \beta}(\widetilde{X}, n)=\sum_{k=1}^{n} \sum_{\tilde{\phi}^{d}:(-n, 1) \nearrow(k-n-1, m)} \int_{\tilde{\phi}^{s d}:(k-n-1, m) \nearrow(-1, \tilde{X})} e^{E(\widetilde{\phi})} \mathrm{d} \widetilde{\phi}^{s d} .
$$

The Brownian motions $\widetilde{B}_{k}$ can be thought of as sitting on the vertical rays starting at $(k-n-1, m)$ for $1 \leq k \leq n$ which makes the definitions (2.13)-(2.14) natural.

For $b \in \mathbb{R}^{m}$ and $\beta \in \mathbb{R}^{n}$, let

$$
\mathcal{Z}_{0}^{b, \beta}(X)= \begin{cases}\mathbf{Z}^{b, \beta}(X, m), & \text { if } X>0 \\ \widetilde{\mathbf{Z}}^{\beta, b}(-X, n), & \text { if } X \leq 0\end{cases}
$$

define the $(m, n)$-spiked boundary perturbation for the CDRP, see Fig. 3. Let $\mathcal{Z}^{b, \beta}(T, X)$ denote the partition function of the CDRP with $(m, n)$-spiked boundary perturbation which is the solution of the stochastic heat equation (1.2) with initial condition given by (2.15). Let $\mathcal{F}^{b, \beta}(T, X)=\ln \left(\mathcal{Z}^{b, \beta}(T, X)\right)$ denote the free energy of the CDRP with $(m, n)$-spiked boundary perturbation. Note that for $n=0$, the boundary perturbation (2.15) reduces to the $m$-spiked boundary perturbation considered in [5].

According to the next theorem, the CDRP with $(m, n)$-spiked boundary perturbations is the limit of the O'Connell-Yor semi-discrete directed polymer with log-gamma boundary sources under the intermediate disorder scaling. The theorem in this form was not published yet, and it was first announced in [11] for the O'Connell-Yor semi-discrete directed polymer with boundary perturbations and used, e.g. in [5,6]. Theorem 2.4 for perturbed boundaries below is a straightforward consequence of the ones used in [5,6]. Intermediate disorder scaling results were, however, proved more recently for the unperturbed multi-layer semi-discrete directed polymer in [16] using the same ideas as in [11]. 


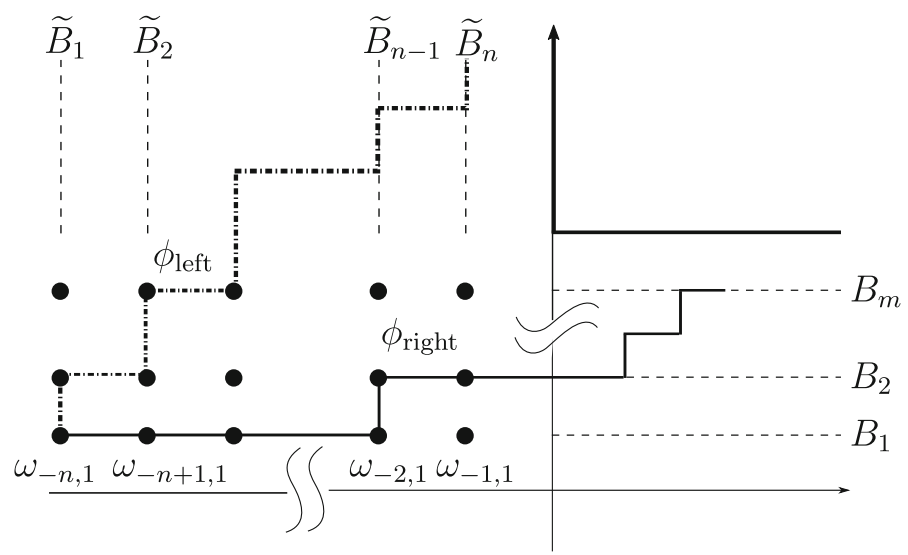

Fig. $3(m, n)$-spiked boundary perturbation $\mathcal{Z}_{0}^{b, \beta}$ for the CDRP, i.e. the $(m, n)$-spiked initial condition for the stochastic heat equation. It is realized by two semi-discrete polymer partition functions with log-gamma boundary sources where the log-gamma random variables are sampled jointly. For $X>0, \mathbf{Z}^{b, \beta}(X, m)$ appears on the horizontal half-line starting at $(0, m+1)$, whereas $\widetilde{\mathbf{Z}}^{\beta, b}(-X, n)$ for $X \leq 0$ appears on the vertical half-line starting at the same point

Theorem 2.4 Fix $T>0, X \in \mathbb{R}$ and real vectors $b=\left(b_{1}, \ldots, b_{m}\right) \in \mathbb{R}^{m}$ and $\beta=\left(\beta_{1}, \ldots \beta_{n}\right) \in \mathbb{R}^{n}$ which satisfy (2.6). Set $\sigma=(2 / T)^{1 / 3}$ and $\kappa=\sqrt{T / N}+X / N$ which yield by (2.10) that $\tau=\kappa N=\sqrt{T N}+X$. Let the drifts be given by $a=$ $\left(a_{1}, \ldots, a_{m}, 0, \ldots, 0\right) \in \mathbb{R}^{N}$ where $a_{l}=\sqrt{N / T}+1 / 2+b_{l}$ for $1 \leq l \leq m$ and the boundary parameters by $\alpha_{k}=\sqrt{N / T}+1 / 2+\beta_{k}$ for $1 \leq k \leq n$. Consider the $O$ 'Connell-Yor semi-discrete directed random polymer partition function $\mathbf{Z}^{a, \alpha}(\tau, N)$ defined in (2.2) with parameters $a$ and $\alpha$. With the scaling factor

$$
C(N, m, T, X)=\exp \left(\frac{1}{2}(N-m) \ln \left(\frac{T}{N}\right)+N+\frac{1}{2}(\sqrt{T N}+X)+X \sqrt{\frac{N}{T}}\right),
$$

one has the convergence in distribution

$$
\frac{\mathbf{Z}^{a, \alpha}(\sqrt{T N}+X, N)}{C(N, m, T, X)} \Rightarrow \mathcal{Z}^{b, \beta}(T, X)
$$

as $N$ goes to infinity where $\mathcal{Z}^{b, \beta}(T, X)$ is the CDRP with $(m, n)$-spiked boundary perturbation given in (2.15).

The main contribution of this work gives the large time limit of the CDRP free energy with $(m, n)$-spiked boundary perturbation.

Theorem 2.5 Let $b=\left(b_{1}, \ldots, b_{m}\right) \in \mathbb{R}^{m}$ and $\beta=\left(\beta_{1}, \ldots \beta_{n}\right) \in \mathbb{R}^{n}$ be such that $b_{l}<\beta_{k}$ for all $1 \leq l \leq m$ and $1 \leq k \leq n$. Let $\sigma=(2 / T)^{1 / 3}$ be scaled with the time parameter and let $Y \in \mathbb{R}$ and $r \in \mathbb{R}$ be arbitrary. Then, for the free energy of the CDRP with $(m, n)$-spiked boundary perturbation of parameters $\sigma b$ and $\sigma \beta$ at rescaled position $X=2^{1 / 3} Y T^{2 / 3}$, 


$$
\lim _{T \rightarrow \infty} \mathbb{P}\left(\frac{\mathcal{F}^{\sigma b, \sigma \beta}\left(T, 2^{1 / 3} Y T^{2 / 3}\right)+T / 24}{(T / 2)^{1 / 3}} \leq r\right)=F_{\mathrm{BP}, b+Y, \beta+Y}\left(r+Y^{2}\right)
$$

holds where $F_{\mathrm{BP}, b+Y, \beta+Y}$ is the Borodin-Péché distribution function given by (2.7) with parameter vectors shifted coordinatewise.

\section{Scaling Limit for the O'Connell-Yor Semi-discrete Polymer}

We prove Theorem 2.3 in this section which is a modification of the proof of Theorem 1.3 in [5]. We mention that in Theorem 1.3 in [5] which is the $n=0$ case of Theorem 2.3, the factor $c_{\kappa}$ is missing in the scaling of parameters (2.11). To keep our discussion self-contained, we recall the main steps of the proof and extend it to the present setup.

Let us scale

$$
u=u(N, r, \kappa)=\exp \left(-N f_{\kappa}-r c_{\kappa} N^{1 / 3}\right)
$$

and set $\tau=\kappa N$. After the change of variables $\tilde{z}=s+v$ in (2.5) and by using Euler's reflection formula $1 /(\Gamma(-s) \Gamma(1+s))=-\pi / \sin (\pi s)$,

$$
\begin{aligned}
\mathrm{K}_{u}\left(v, v^{\prime}\right)= & \frac{-1}{2 \pi \mathrm{i}} \int_{\mathcal{C}_{\tilde{z}}} \mathrm{~d} \tilde{z} \frac{\pi}{\sin (\pi(\tilde{z}-v))} \frac{\exp \left(N G(v)+r c_{\kappa} N^{1 / 3} v\right)}{\exp \left(N G(\tilde{z})+r c_{\kappa} N^{1 / 3} \tilde{z}\right)} \frac{1}{\tilde{z}-v^{\prime}} \\
& \times \prod_{l=1}^{m} \frac{\Gamma\left(v-a_{l}\right) \Gamma(\tilde{z})}{\Gamma\left(\tilde{z}-a_{l}\right) \Gamma(v)} \prod_{k=1}^{n} \frac{\Gamma\left(\alpha_{k}-v-s\right)}{\Gamma\left(\alpha_{k}-v\right)}
\end{aligned}
$$

where $G(z)=\ln \Gamma(z)-\kappa z^{2} / 2+f_{\kappa} z$. The integration contour $\mathcal{C}_{\tilde{z}}$ in (3.2) was defined in [5] in the absence of boundary parameters to be

$$
\left\{\theta_{\kappa}+\tilde{\varepsilon}+\mathrm{i} y, y \in \mathbb{R}\right\} \cup \bigcup_{q=1}^{r} B_{v+q}
$$

where $B_{v+q}$ denotes a small circle around $v+q$ and clockwise oriented. $r \in \mathbb{N}_{0}$ is chosen such that $\operatorname{Re}(v)+r \leq \theta_{\kappa}+\mathcal{O}\left(N^{-1 / 3}\right)$ and we set $\tilde{\varepsilon}=p(v) c_{\kappa}^{-1} N^{-1 / 3}$ with $p(v) \in\{1,3\}$. This choice of $r$ and $p(v)$ is needed to keep a uniformly positive distance from the poles coming from the sine in the denominator in (3.2), see Sect. 5.1 in [5] for the precise definition. It is also argued in [5] that kernel $K_{u}$ has enough decay along the contour in (3.3) which corresponds to the $\varphi=\pi / 4$ case for the $\mathcal{C}_{a ; \alpha ; \varphi}$ contour in Theorem 2.1.

In the present setup when there are boundary parameters $a_{l}$ and $\alpha_{k}$ scaled according to (2.11), the contour $\mathcal{C}_{\tilde{z}}$ is defined to be the contour (3.3) with a local modification in an $N^{-1 / 3}$ neighbourhood of $\theta_{\kappa}$ in a way that it crosses the real axis between the $a_{l}$ 
Fig. 4 Integration paths $\mathcal{C}_{w}$ and $\mathcal{C}_{z}$. The black dots on the left are the values of $b_{1}, \ldots, b_{m}$ and the grey dots on the right are $\beta_{1}, \ldots, \beta_{n}$

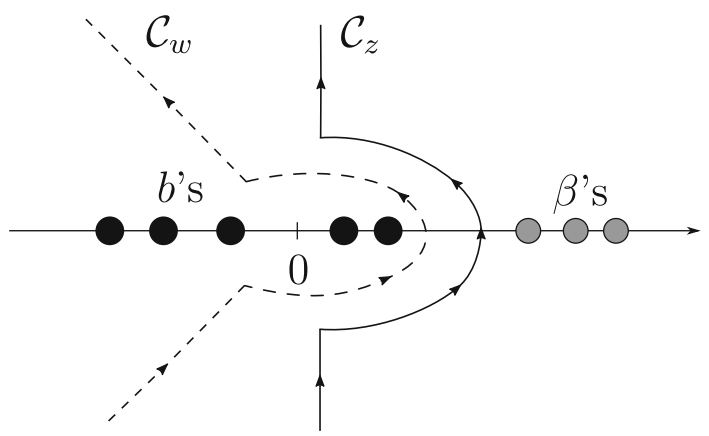

and the $\alpha_{k}$ singularities. By the Cauchy theorem, the contour $v+\mathcal{D}_{v}$ for $\tilde{z}$ seen on the left of Fig. 2 can be replaced by $\mathcal{C}_{\tilde{z}}$ without changing the kernel $\mathrm{K}_{u}$ in (3.2).

The function $G$ has a double critical point at $\theta_{\kappa}$, i.e. $G(v) \simeq G\left(\theta_{\kappa}\right)-\frac{\left(c_{\kappa}\right)^{3}}{3}\left(v-\theta_{\kappa}\right)^{3}$. This suggests the rescaling around $\theta_{\kappa}$ by $N^{1 / 3}$, that is the change of variables

$$
\left\{v, v^{\prime}, \tilde{z}\right\}=\left\{\Phi(w), \Phi\left(w^{\prime}\right), \Phi(z)\right\} \quad \text { with } \Phi(z)=\theta_{\kappa}+z c_{\kappa}^{-1} N^{-1 / 3} .
$$

Then, the rescaled kernel is defined as

$$
\begin{aligned}
\mathrm{K}_{N}\left(w, w^{\prime}\right)= & c_{\kappa}^{-1} N^{-1 / 3} \mathrm{~K}_{u}\left(\Phi(w), \Phi\left(w^{\prime}\right)\right)=\frac{-c_{\kappa}^{-1} N^{-1 / 3}}{2 \pi \mathrm{i}} \\
& \times \int_{\Phi^{-1}\left(\mathcal{C}_{\tilde{z}}\right)} \mathrm{d} z \frac{\pi e^{N G(\Phi(w))-N G(\Phi(z))}}{\sin \left(\pi(z-w) c_{\kappa}^{-1} N^{-1 / 3}\right)} \\
& \times \frac{e^{r(w-z)}}{z-w^{\prime}} \prod_{l=1}^{m} \frac{\Gamma\left(\Phi(w)-a_{l}\right) \Gamma(\Phi(z))}{\Gamma\left(\Phi(z)-a_{l}\right) \Gamma(\Phi(w))} \prod_{k=1}^{n} \frac{\Gamma\left(\alpha_{k}-\Phi(z)\right)}{\Gamma\left(\alpha_{k}-\Phi(w)\right)} .
\end{aligned}
$$

Let the new contour $\mathcal{C}_{w}$ be the local perturbation of $\{-|y|+\mathrm{i} y, y \in \mathbb{R}\}$ in a constant neighbourhood of 0 in a way that it crosses the real axis between the $b_{l}$ and $\beta_{k}$ singularities as shown in Fig. 4, also compare with Fig. 2. Further, let $\mathcal{C}_{z}$ be the local modification of $1+i \mathbb{R}$ in a neighbourhood of 0 so that it does not intersect $\mathcal{C}_{w}$, and it crosses the real axis between the two families of singularities. Then, one can replace $\mathcal{C}_{a ; \alpha ; \varphi}$ by $\mathcal{C}_{w}$ and the integration path $\Phi^{-1}\left(\mathcal{C}_{\tilde{z}}\right)$ in $(3.5)$ by $\mathcal{C}_{z}$ so that one has the equality of Fredholm determinants $\operatorname{det}\left(\mathbb{1}+\mathrm{K}_{u}\right)_{L^{2}\left(\mathcal{C}_{a ; \alpha ; \varphi}\right)}=\operatorname{det}\left(\mathbb{1}+\mathrm{K}_{N}\right)_{L^{2}\left(\mathcal{C}_{w}\right)}$ by the Cauchy theorem.

Based on the next two propositions and by using Lemma 3.3, Theorem 2.3 on the scaling limit for the O'Connell-Yor semi-discrete polymer can be verified.

Proposition 3.1 Let $\mathrm{K}_{N}\left(w, w^{\prime}\right)$ be given in (3.5). Uniformly for $w$, $w^{\prime}$ in a bounded set of $\mathcal{C}_{w}$,

$$
\lim _{N \rightarrow \infty} \mathrm{K}_{N}\left(w, w^{\prime}\right)=\widetilde{\mathrm{K}}_{\mathrm{BP}, b, \beta}\left(w, w^{\prime}\right)
$$


where

$$
\widetilde{\mathrm{K}}_{\mathrm{BP}, b, \beta}\left(w, w^{\prime}\right):=\frac{1}{2 \pi \mathrm{i}} \int_{\mathcal{C}_{z}} \mathrm{~d} z \frac{1}{(w-z)\left(z-w^{\prime}\right)} \frac{e^{z^{3} / 3-r z}}{e^{w^{3} / 3-r w}} \prod_{l=1}^{m} \frac{z-b_{l}}{w-b_{l}} \prod_{k=1}^{n} \frac{w-\beta_{k}}{z-\beta_{k}} .
$$

Proposition 3.2 For any $w, w^{\prime} \in \mathcal{C}_{w}$ there exists a constant $C \in(0, \infty)$ such that

$$
\left|\mathrm{K}_{N}\left(w, w^{\prime}\right)\right| \leq C e^{-|\operatorname{Im}(w)|}
$$

uniformly for all $N$ large enough.

Lemma 3.3 [4, Lemma 4.1.38] Consider a sequence of functions $\left(\Theta_{n}\right)_{n \geq 1}$ mapping $\mathbb{R} \rightarrow[0,1]$ with the following properties: $x \mapsto \Theta_{n}(x)$ is strictly decreasing, $\lim _{x \rightarrow-\infty} \Theta_{n}(x)=1, \lim _{x \rightarrow \infty} \Theta_{n}(x)=0$ for all $n$ and $\Theta_{n}(x) \rightarrow \mathbb{1}_{x \leq 0}$ as $n \rightarrow \infty$ uniformly on $\mathbb{R} \backslash[-\delta, \delta]$ for all $\delta>0$. Consider a sequence of random variables $X_{n}$ and a continuous probability distribution function $p(r)$ such that $\mathbb{E}\left[\Theta_{n}\left(X_{n}-r\right)\right] \rightarrow p(r)$ as $n \rightarrow \infty$ for each $r \in \mathbb{R}$. Then, $X_{n}$ converges in distribution to the distribution given by $p(r)$.

Proof of Theorem 2.3 By Hadamard's bound and by dominated convergence, Propositions 3.1 and 3.2 together imply that

$$
\begin{gathered}
\operatorname{det}\left(\mathbb{1}+\mathrm{K}_{N}\right)_{L^{2}\left(\mathcal{C}_{w}\right)} \rightarrow \operatorname{det}\left(\mathbb{1}-\widetilde{\mathrm{K}}_{\mathrm{BP}, b, \beta}\right)_{L^{2}\left(\mathcal{C}_{w}\right)} \\
=\operatorname{det}\left(\mathbb{1}-\mathrm{K}_{\mathrm{BP}, b, \beta}\right)_{L^{2}((r, \infty))}=F_{\mathrm{BP}, b, \beta}(r)
\end{gathered}
$$

as $N \rightarrow \infty$ where the first equality above follows from the same reformulation of Fredholm determinants as in Lemma 8.7 of [5].

Let us define a sequence of functions $\Theta_{N}(x)=\exp \left(-\exp \left(c_{\kappa} N^{1 / 3} x\right)\right)$. Now by (3.1),

$$
\begin{aligned}
\mathbb{E} & {\left[\Theta_{N}\left(\frac{F^{a, \alpha}(\kappa N)-N f^{\kappa}}{c_{\kappa} N^{1 / 3}}-r\right)\right] } \\
& =\mathbb{E}\left[e^{u \mathbf{Z}^{a, \alpha}(\tau, N)}\right]=\operatorname{det}\left(\mathbb{1}+\mathrm{K}_{u}\right)_{L^{2}\left(\mathcal{C}_{a ; \alpha ; \varphi}\right)} \rightarrow F_{\mathrm{BP}, b, \beta}(r)
\end{aligned}
$$

as $N \rightarrow \infty$ where we used the definition of $\Theta_{N}$, Theorem 2.1 and (3.9). To conclude the proof, one uses Lemma 3.3 with $p(r)=F_{\mathrm{BP}, b, \beta}(r)$.

We introduce the extra gamma factors

$$
P(w, z, a)=\frac{\Gamma\left(\Phi(w)-a_{l}\right)}{\Gamma(\Phi(w))} \frac{\Gamma(\Phi(z))}{\Gamma\left(\Phi(z)-a_{l}\right)}, Q\left(w, z, \alpha_{k}\right)=\frac{\Gamma\left(\alpha_{k}-\Phi(z)\right)}{\Gamma\left(\alpha_{k}-\Phi(w)\right)}
$$

for $1 \leq l \leq m$ and $1 \leq k \leq n$. To extend the proofs of Propositions 5.1 and 5.2 of [5] to those of Propositions 3.1 and 3.2, the following lemma about the bounds on the extra factors is the key. 
Lemma 3.4 Once the contours $\mathcal{C}_{w}$ and $\mathcal{C}_{z}$ are fixed, there is a constant $C$ such that

$$
\left|Q\left(w, z, \alpha_{k}\right)\right| \leq C \frac{|w|}{N^{1 / 3}}\left(1+\frac{N^{1 / 3}}{|z|}\right) \leq C|w|\left(1+\frac{1}{|z|}\right)
$$

as long as $w \in \mathcal{C}_{w}$ and $z \in \mathcal{C}_{z}$.

Furthermore, let $N$ be large enough to make the $N^{-1 / 3}$ difference of $\mathcal{C}_{\tilde{z}}$ and the contour in (3.3) small. Then, the small circles in $\mathcal{C}_{\tilde{z}}$ and in (3.3) can only be present, i.e. $r>0$ can only happen for a $v \in \mathcal{C}_{a ; \alpha ; \varphi}$ if $|v|>\varepsilon$ for some fixed $\varepsilon>0$. In this case, there is a $C$ such that for any $v \in \mathcal{C}_{a ; \alpha ; \varphi}$ and $q=1, \ldots, r$,

$$
\left|Q\left(\Phi^{-1}(v), \Phi^{-1}(v+q), \alpha_{k}\right)\right| \leq C
$$

Proof After substituting (3.4) and the scaling (2.11) into the definition (3.11), one can write

$$
Q(w, z, \alpha)=\frac{\Gamma\left(\left(\beta_{k}-z+o(1)\right) c_{\kappa}^{-1} N^{-1 / 3}\right)}{\Gamma\left(\left(\beta_{k}-w+o(1)\right) c_{\kappa}^{-1} N^{-1 / 3}\right)} .
$$

First, we show that for the numerator,

$$
\left|\Gamma\left(\left(\beta_{k}-z+o(1)\right) c_{\kappa}^{-1} N^{-1 / 3}\right)\right| \leq C\left(1+\frac{N^{1 / 3}}{|z|}\right)
$$

holds if $z \in \mathcal{C}_{z}$. To this end, we use the asymptotics

$$
\lim _{|y| \rightarrow \infty}|\Gamma(x+i y)|(2 \pi)^{-1 / 2} e^{\frac{1}{2} \pi|y|}|y|^{\frac{1}{2}-x}=1
$$

from equation 6.1 .45 of [1]. If $z \in \mathcal{C}_{z}$ and $|z|>\delta N^{1 / 3}$ for some fixed $\delta>0$, then the real part of the argument of the gamma function in (3.15) goes to 0 as $N \rightarrow \infty$; hence, it is bounded. Consequently, (3.16) yields an exponential decay of $\left|\Gamma\left(\left(\beta_{k}-z+o(1)\right) c_{\kappa}^{-1} N^{-1 / 3}\right)\right|$ in $|z|$ which we bound by a constant. By the asymptotics $\Gamma(Z) \sim 1 / Z$ around $Z=0$, one gets that the left-hand side of (3.15) can be upper bounded by $C N^{1 / 3} /|z|$ as long as $|z|<\delta N^{1 / 3}$. This proves (3.15).

Next, we prove for the denominator that

$$
\left|\Gamma\left(\left(\beta_{k}-w+o(1)\right) c_{\kappa}^{-1} N^{-1 / 3}\right)\right| \geq c\left(1+\frac{N^{1 / 3}}{|w|}\right)
$$

for $w \in \mathcal{C}_{w}$ with a constant $c$ small enough. Equation 6.1.37 in [1] reads as

$$
\Gamma(z)=e^{-z} z^{z-\frac{1}{2}}(2 \pi)^{1 / 2}\left(1+\mathcal{O}\left(\frac{1}{z}\right)\right) .
$$


For $w \in \mathcal{C}_{w}$ and $|w|>\delta N^{1 / 3}$, one can write $w=-t N^{1 / 3} \pm \mathrm{i} t N^{1 / 3}$ for some $t>\delta / \sqrt{2}$. Hence, the left-hand side of (3.17) grows as $C e^{-t} t^{t}$ as $t \rightarrow \infty$ which we can lower bound by a small constant as $t>\delta / \sqrt{2}$. If $|w|<\delta N^{1 / 3}$, by the asymptotics $\Gamma(Z) \sim 1 / Z$ around $Z=0$ again, the left-hand side of (3.17) is lower bounded by $c N^{1 / 3} /|w|$. This shows (3.17). Putting (3.15) and (3.17) together yields (3.12) with a large enough $C$.

The uniform lower bound on $|v|$ follows from the choice of the contours. On the one hand, $r$ is chosen such that $\operatorname{Re}(v)+r \leq \theta_{\kappa}+\mathcal{O}\left(N^{-1 / 3}\right)$. On the other hand, $v \in \mathcal{C}_{a ; \alpha ; \varphi}$ satisfies $v=\theta_{\kappa}+\mathcal{O}\left(N^{-1 / 3}\right)+e^{\mathrm{i}(\pi \pm \varphi)} y$ for some $y \in \mathbb{R}_{+}$. These two properties imply the lower bound if the small circles are present.

To show (3.13) if the circles are present, observe that the ratio which we want to bound in absolute value simplifies as

$$
Q\left(\Phi^{-1}(v), \Phi^{-1}(v+q), \alpha_{k}\right)=\frac{\Gamma\left(\alpha_{k}-v-q\right)}{\Gamma\left(\alpha_{k}-v\right)}=\frac{1}{\left(\alpha_{k}-v-1\right) \ldots\left(\alpha_{k}-v-q\right)} .
$$

This is bounded by an absolute constant since $|v|>\varepsilon$ also means that $|\operatorname{Im}(v)|$ is uniformly positive.

Proof of Proposition 3.1 Knowing the asymptotics $\Gamma(z) \simeq 1 / z$ near zero from [1], one can conclude from (3.14) that under the scaling (2.11), $Q\left(w, z, \alpha_{k}\right) \rightarrow\left(w-\beta_{k}\right) /(z-$ $\beta_{k}$ ) holds as $N \rightarrow \infty$ for $k=1, \ldots, n$. Similarly, $P\left(w, z, a_{l}\right) \rightarrow\left(z-b_{l}\right) /\left(w-b_{l}\right)$ for $l=1, \ldots, m$. As in the proof of Proposition 5.1 in [5], the Taylor expansion of the remaining factors in the integrand of $\mathrm{K}_{N}$ in (3.5) yields that the integrand converges to the integrand of $\widetilde{\mathrm{K}}_{\mathrm{BP}, b, \beta}$ in (3.7).

One can apply dominated convergence as it is done in the proof of Proposition 5.1 in [5]. It was proved in [5] based on Lemma 5.4 that the integration contour of $\mathrm{K}_{N}$ in $z$ is steep descent for the function $-\operatorname{Re}(G(\Phi(z)))$ with derivative going to $-\infty$ linearly in $|\operatorname{Im}(\tilde{z})|=N^{-1 / 3}|\operatorname{Im}(z)|$. Since the $Q$ factors are bounded in (3.12), the decay of $e^{-N G(\Phi(z))}$ ensures that the integral which defines the kernel $K_{N}$ in (3.5) is still convergent in the presence of the $Q$ factors. Hence, the steps of the proof of Proposition 5.1 in [5] can be followed. In particular, the integral which defines $\mathrm{K}_{N}$ restricted to the set $|\operatorname{Im}(z)|>\delta N^{1 / 3}$ is $\mathcal{O}\left(e^{-c(\delta) N}\right)$. On the other hand, on $|\operatorname{Im}(z)|<\delta N^{1 / 3}$, one can replace the integrand of $\mathrm{K}_{N}$ by the integrand of $\widetilde{\mathrm{K}}_{\mathrm{BP}, b, \beta}$ with an overall error of order $\mathcal{O}\left(N^{-1 / 3}\right)$. This verifies the convergence of the kernels (3.6).

Proof of Proposition 3.2 The exponential bounds obtained in the proof of Proposition 5.2 in [5] are not affected by the presence of extra polynomial factors which upper bound $Q$ in (3.12)-(3.13). Hence (3.8) follows.

\section{Large Time Limit of the CDRP with $(m, n)$-Spiked Boundary Perturbations}

In this section, we prove Theorem 2.5 about the large time limit of the free energy $\mathcal{Z}^{b, \beta}$ of the CDRP with $(m, n)$-spiked boundary perturbations. We start by giving a 
Fredholm determinant formula for its Laplace transform in Proposition 4.1 based on Theorem 2.1. Let $b=\left(b_{1}, \ldots, b_{m}\right)$ and $\beta=\left(\beta_{1}, \ldots, \beta_{n}\right)$ be such that (2.6) holds. Define the kernel

$$
\begin{aligned}
\mathrm{K}_{b, \beta}^{(\sigma)}(x, y)= & \frac{1}{(2 \pi \mathrm{i})^{2}} \int \mathrm{d} w \int \mathrm{d} z \frac{\sigma \pi S^{\sigma(z-w)}}{\sin (\sigma \pi(z-w))} \frac{e^{z^{3} / 3-z y}}{e^{w^{3} / 3-w x}} \prod_{l=1}^{m} \frac{\Gamma\left(\sigma w-b_{l}\right)}{\Gamma\left(\sigma z-b_{l}\right)} \\
& \times \prod_{k=1}^{n} \frac{\Gamma\left(\beta_{k}-\sigma z\right)}{\Gamma\left(\beta_{k}-\sigma w\right)}
\end{aligned}
$$

where

$$
\sigma=(2 / T)^{1 / 3}
$$

and the integration contour for $w$ is from $-\frac{1}{4 \sigma}-\mathrm{i} \infty$ to $-\frac{1}{4 \sigma}+\mathrm{i} \infty$ and crosses the real axis between $\max _{1 \leq l \leq m} b_{l} / \sigma$ and $\min _{1 \leq k \leq n} \beta_{k} / \sigma$. The other contour for $z$ goes from $\frac{1}{4 \sigma}-\mathrm{i} \infty$ to $\frac{1}{4 \sigma}+\mathrm{i} \infty$, it also crosses the real axis between $\max _{1 \leq l \leq m} b_{l} / \sigma$ and $\min _{1 \leq k \leq n} \beta_{k} / \sigma$, and it does not intersect the contour for $w$.

Proposition 4.1 Fix $S$ with positive real part, $T>0, b$ and $\beta$ real vectors with (2.6). Set $\sigma$ as in (4.2). Then,

$$
\mathbb{E}\left[\exp \left(-\operatorname{Se}^{\frac{X^{2}}{2 T}+\frac{T}{24}} \mathcal{Z}^{b, \beta}(T, X)\right)\right]=\operatorname{det}\left(\mathbb{1}-\mathrm{K}_{b+X / T, \beta+X / T}^{(\sigma)}\right)_{L^{2}\left(\mathbb{R}_{+}\right)}
$$

where $\mathcal{Z}^{b, \beta}$ is the partition function of the CDRP with $(m, n)$-spiked boundary perturbations and $\mathrm{K}_{b, \beta}^{(\sigma)}$ is defined in (4.1).

Proof Let Theorem 2.1 be used with

$$
u=\frac{S}{C(N, m, T, X)} e^{\frac{X^{2}}{2 T}+\frac{T}{24}}
$$

where $C(N, m, T, X)$ is given by (2.16). Then, on the left-hand side of (2.4) with $\tau=$ $\sqrt{T N}+X$, Theorem 2.4 on the intermediate disorder scaling yields the convergence in distribution

$$
u \mathbf{Z}^{a, \alpha}(\sqrt{T N}+X, N) \Rightarrow S e^{\frac{X^{2}}{2 T}+\frac{T}{24}} \mathcal{Z}^{b, \beta}(T, X)
$$

as $N \rightarrow \infty$. By definition (2.2), the partition function $\mathbf{Z}^{a, \alpha}$ is positive; hence, (4.5) implies the convergence of the Laplace transforms

$$
\mathbb{E}\left[e^{-u \mathbf{Z}^{a, \alpha}(\tau, N)}\right] \rightarrow \mathbb{E}\left[\exp \left(-S e^{\frac{X^{2}}{2 T}+\frac{T}{24}} \mathcal{Z}^{b, \beta}(T, X)\right)\right]
$$

as $N \rightarrow \infty$ where $\tau=\sqrt{T N}+X$ and $u$ is defined in (4.4). 
On the other hand, the same scaling of parameters is used on the right-hand side of (2.4). Then, Theorem 6.3 of [6] is used to conclude the convergence of Fredholm determinants

$$
\lim _{N \rightarrow \infty} \operatorname{det}\left(\mathbb{1}+\mathrm{K}_{u}\right)_{L^{2}\left(\mathcal{C}_{a ; \alpha ; \pi / 4}\right)}=\operatorname{det}\left(\mathbb{1}-\mathrm{K}_{b+X / T, \beta+X / T}^{(\sigma)}\right)_{L^{2}\left(\mathbb{R}_{+}\right)}
$$

under the following scaling of the parameters. As in Theorem 2.4, one sets $\tau=$ $\sqrt{T N}+X, \kappa=\tau / N$ and $\theta_{\kappa}$ is given by (2.10). This means that $\theta_{\kappa}=\sqrt{N / T}-$ $X / T+1 / 2+\mathcal{O}\left(N^{-1 / 2}\right)$. One sets $u$ given by (4.4) and $\sigma$ given by (4.2). For the boundary parameters $a_{l}$ and $\alpha_{k}$, instead of the scaling given in Theorem 2.4, one sets $a_{l}=\theta_{\kappa}+b_{l}$ and $\alpha_{k}=\theta_{\kappa}+\beta_{k}$ according to Sect. 6 of [6]. This difference results in the shift by $X / T$ in the rescaled boundary parameters $b_{l}$ and $\beta_{k}$ which completes the proof.

The following proposition is the key for the proof of Theorem 2.5.

Proposition 4.2 We have

$$
\operatorname{det}\left(\mathbb{1}-\mathrm{K}_{\sigma b, \sigma \beta}^{(\sigma)}\right)_{L^{2}\left(\mathbb{R}_{+}\right)} \rightarrow \operatorname{det}\left(\mathbb{1}-\mathrm{K}_{B P, b, \beta}\right)_{L^{2}(r, \infty)}
$$

as $\sigma \rightarrow 0$ where $\mathrm{K}_{b, \beta}^{(\sigma)}$ and $\mathrm{K}_{B P, b, \beta}$ are given in (4.1) and (2.8).

Proof of Theorem 2.5 Let $S=e^{-r / \sigma}$ and define the functions $\Theta_{T}(x)=\exp \left(-e^{x / \sigma}\right)$ where $\sigma=(2 / T)^{1 / 3}$. Observe that one can write

$$
\exp \left(-\operatorname{Se}^{\frac{X^{2}}{2 T}+\frac{T}{24}} \mathcal{Z}^{b, \beta}(T, X)\right)=\Theta_{T}\left(\frac{\mathcal{F}^{\sigma b, \sigma \beta}(T, X)+\frac{X^{2}}{2 T}+\frac{T}{24}}{\sigma^{-1}}-r\right)
$$

By taking expectation above

$$
\begin{aligned}
\mathbb{E}\left[\Theta_{T}\left(\frac{\mathcal{F}^{\sigma b, \sigma \beta}(T, X)+\frac{X^{2}}{2 T}+\frac{T}{24}}{\sigma^{-1}}-r\right)\right] & =\mathbb{E}\left[\exp \left(-S^{\frac{X^{2}}{2 T}+\frac{T}{24}} \mathcal{Z}^{b, \beta}(T, X)\right)\right] \\
& =\operatorname{det}\left(\mathbb{1}-\mathrm{K}_{\sigma b+X / T, \sigma \beta+X / T}^{(\sigma)}\right)_{L^{2}\left(\mathbb{R}_{+}\right)} \\
& \rightarrow \operatorname{det}\left(\mathbb{1}-\mathrm{K}_{B P, b+Y, \beta+Y}\right)_{L^{2}(r, \infty)}
\end{aligned}
$$

as $T \rightarrow \infty$ where we used Proposition 4.1 in the second equation above. To conclude the convergence in (4.10), Proposition 4.2 was used with boundary parameters $\sigma b+$ $X / T=\sigma(b+Y)$ and $\sigma \beta+X / T=\sigma(\beta+Y)$ where $X=2^{1 / 3} Y T^{2 / 3}$.

The functions $\Theta_{T}$ satisfy the properties of Lemma 3.3; hence, by (4.10) the lemma is applicable for the random variables $\sigma\left(\mathcal{F}^{\sigma b, \sigma \beta}(T, X)+X^{2} /(2 T)+T / 24\right)$ and with the distribution function $F_{\mathrm{BP}, b+Y, \beta+Y}(r)$ defined by (2.7). By observing that $\sigma X^{2} /(2 T)=Y^{2}$ and by substituting $r$ by $r+Y^{2}$, one arrives to (2.18). 
We are left with proving Proposition 4.2. We use the following decay bound from [6] adapted to the present setting.

Lemma 4.3 [6, Lemma B.4] Fix $b_{1} \leq b_{2} \leq \cdots \leq b_{m}<\beta_{1} \leq \beta_{2} \cdots \leq \beta_{n}$ so that $\beta_{i}-b_{j}<1$ for any $1 \leq i \leq n$ and $1 \leq j \leq m$. Then, there is a finite constant $C$ such that for any $x, y \in \mathbb{R}_{+}$

$$
\left|\mathrm{K}_{b, \beta}^{(\sigma)}(x, y)\right| \leq C \exp \left(-\frac{\beta_{1}}{\sigma} y+\frac{b_{m}}{\sigma} x\right)
$$

Proof of Proposition 4.2 By setting $S=e^{-r / \sigma}$, the kernel on the left-hand side of (4.8) reads as

$$
\begin{aligned}
\mathrm{K}_{\sigma b, \sigma \beta}^{(\sigma)}(x, y)= & \frac{1}{(2 \pi \mathrm{i})^{2}} \int \mathrm{d} w \int \mathrm{d} z \frac{\sigma \pi e^{-r(z-w)}}{\sin (\sigma \pi(z-w))} \frac{e^{z^{3} / 3-z y}}{e^{w^{3} / 3-w x}} \prod_{l=1}^{m} \frac{\Gamma\left(\sigma\left(w-b_{l}\right)\right)}{\Gamma\left(\sigma\left(z-b_{l}\right)\right)} \\
& \times \prod_{k=1}^{n} \frac{\Gamma\left(\sigma\left(\beta_{k}-z\right)\right)}{\Gamma\left(\sigma\left(\beta_{k}-w\right)\right)} .
\end{aligned}
$$

Then, the first factor in the double integral in (4.12) converges to $e^{-r(z-w)} /(z-w)$ as $\sigma \rightarrow 0$. For the product of the gamma ratios,

$$
\prod_{l=1}^{m} \frac{\Gamma\left(\sigma\left(w-b_{l}\right)\right)}{\Gamma\left(\sigma\left(z-b_{l}\right)\right)} \prod_{k=1}^{n} \frac{\Gamma\left(\sigma\left(\beta_{k}-z\right)\right)}{\Gamma\left(\sigma\left(\beta_{k}-w\right)\right)} \rightarrow \prod_{l=1}^{m} \frac{z-b_{l}}{w-b_{l}} \prod_{k=1}^{n} \frac{w-\beta_{k}}{z-\beta_{k}}
$$

as $\sigma \rightarrow 0$. Hence, the integrand in (4.12) converges to that of $\mathrm{K}_{\mathrm{BP}, b, \beta}(x+r, y+r)$ given in (2.8) as $\sigma \rightarrow 0$. Since along the contours for $w$ and $z$, the factors $e^{z^{3} / 3-w^{3} / 3}$ in (4.12) have fast enough decay, we conclude that

$$
\lim _{\sigma \rightarrow 0} \mathrm{~K}_{\sigma b, \sigma \beta}^{(\sigma)}(x, y)=\mathrm{K}_{\mathrm{BP}, b, \beta}(x+r, y+r) .
$$

To show that the convergence of the kernels (4.14) implies the convergence of Fredholm determinants (4.8), one uses dominated convergence. Lemma 4.3 applied to $\mathrm{K}_{\sigma b, \sigma \beta}^{(\sigma)}$ provides a uniform upper bound in $\sigma$. Using this upper bound, the $n$th term in the Fredholm determinant expansion of the left-hand side of (4.8) is bounded by

$$
\begin{aligned}
& \frac{1}{n !} \int_{\mathbb{R}_{+}} \ldots \int_{\mathbb{R}_{+}} \operatorname{det}\left[\mathrm{K}_{\sigma b, \sigma \beta}^{(\sigma)}\left(x_{i}, x_{j}\right)\right]_{i, j=1}^{n} \mathrm{~d} x_{1} \ldots \mathrm{d} x_{n} \\
& \quad \leq \frac{C^{2 n} n^{n / 2}}{n !} \int_{\mathbb{R}_{+}} \ldots \int_{\mathbb{R}_{+}} e^{-\left(\beta_{1}-b_{m}\right) \sum_{j=1}^{n} x_{j}} \mathrm{~d} x_{1} \ldots \mathrm{d} x_{n} \\
& \quad=\frac{C^{2 n} n^{n / 2}}{\left(\beta_{1}-b_{m}\right)^{n} n !}
\end{aligned}
$$


where we also used the Hadamard bound in the first inequality above. Since the righthand side of (4.15) is summable, dominated convergence implies (4.8).

Acknowledgements Open access funding provided by Budapest University of Technology and Economics (BME). We thank Patrik Ferrari and Benedek Valkó for stimulating discussions related to this project. The work of both authors was supported by the NKFI (National Research, Development and Innovation Office) Grant FK123962. The work of B. Vetô was supported by the NKFI Grant PD123994, by the Bolyai Research Scholarship of the Hungarian Academy of Sciences and by the ÚNKP-18-4 New National Excellence Program of the Ministry of Human Capacities.

Open Access This article is distributed under the terms of the Creative Commons Attribution 4.0 International License (http://creativecommons.org/licenses/by/4.0/), which permits unrestricted use, distribution, and reproduction in any medium, provided you give appropriate credit to the original author(s) and the source, provide a link to the Creative Commons license, and indicate if changes were made.

\section{References}

1. Abramowitz, M., Stegun, I.A.: Pocketbook of Mathematical Functions. Verlag Harri Deutsch, Frankfurt a. M. (1984)

2. Baik, J., Ben Arous, G., Péché, S.: Phase transition of the largest eigenvalue for non-null complex sample covariance matrices. Ann. Probab. 33, 1643-1697 (2006)

3. Barraquand, G., Corwin, I.: Random-walk in Beta-distributed random environment. Probab. Theory Relat. Fields 167(3), 1057-1116 (2017)

4. Borodin, A., Corwin, I.: Macdonald processes. Probab. Theory Relat. Fields 158, 225-400 (2014)

5. Borodin, A., Corwin, I., Ferrari, P.L.: Free energy fluctuations for directed polymers in random media in $1+1$ dimension. Commun. Pure Appl. Math. 67, 1129-1214 (2014)

6. Borodin, A., Corwin, I., Ferrari, P.L., Vető, B.: Height fluctuations for the stationary KPZ equation. Math. Phys. Anal. Geom. 18(1), 20 (2015)

7. Borodin, A., Péché, S.: Airy kernel with two sets of parameters in directed percolation and random matrix theory. J. Stat. Phys. 132(2), 275-290 (2008)

8. Corwin, I.: The Kardar-Parisi-Zhang equation and universality class. Random Matices Theory Appl. 1, 1130001 (2012)

9. Corwin, I., O'Connell, N., Seppäläinen, T., Zygouras, N.: Tropical combinatorics and Whittaker functions. Duke Math. J. 163, 513-563 (2014)

10. Corwin, I., Seppäläinen, T., Shen, H.: The strict-weak lattice polymer. J. Stat. Phys. 160, 1027-1053 (2015)

11. Flores, G. Moreno, Quastel, J., Remenik, D.: In preparation, (2012)

12. Hairer, M.: Solving the KPZ equation. Ann. Math. 178, 559-664 (2013)

13. Imamura, T., Sasamoto, T.: Free energy distribution of the stationary O'connell-Yor directed random polymer model. J. Phys. A 50(28), 285203 (2017)

14. Kardar, M., Parisi, G., Zhang, Y.Z.: Dynamic scaling of growing interfaces. Phys. Rev. Lett. 56, 889892 (1986)

15. Mueller, C.: On the support of solutions to the heat equation with noise. Stoch. Stoch. Rep. 37, 225-246 (1991)

16. Nica, M.: Intermediate disorder limits for multi-layer semi-discrete directed polymers. arXiv:1609.00298 (2016)

17. O'Connell, N.: Directed polymers and the quantum Toda lattice. Ann. Probab. 40, 437-458 (2012)

18. O'Connell, N., Ortmann, J.: Tracy-Widom asymptotics for a random polymer model with gammadistributed weights. Electron. J. Probab. 20, 18 (2015)

19. O’Connell, N., Yor, M.: Brownian analogues of Burke's theorem. Stoch. Proc. Appl. 96, 285-304 (2001)

20. Seppäläinen, T.: Scaling for a one-dimensional directed polymer with boundary conditions. Ann. Probab. 40, 19-73 (2012)

21. Thiery, T., Le Doussal, P.: Log-gamma directed polymer with fixed endpoints via the replica Bethe Ansatz. J. Stat. Mech. Theory Exp. 2014(10), P10018 (2014) 
22. Thiery, T., Le Doussal, P.: On integrable directed polymer models on the square lattice. J. Phys. A 48(46), 465001 (2015)

Publisher's Note Springer Nature remains neutral with regard to jurisdictional claims in published maps and institutional affiliations. 